Niniejsza publikacja jest dostęna na licencji Creative Commons. Uznanie autorstwa-Użycie niekomercyjne-Bez utworów zależnych 3.0 Polska. Pewne prawa zastrzė̇one na rzecz autora. Zezwala się na wykorzystanie publikacji zgodnie z licencja - pod warunkiem zachowania niniejszej informacji licencyjnej oraz wskazania autora jako właściciela praw do tekstu. Treść licencji jest dostępna na stronie: http://creativecommons.org/licenses/by-nc-nd/3.0/pl/

Lingwistyka Stosowana 22: 2/2017, 1-14

\author{
Joanna CHLEBNIKOW \\ Anke BACKHAUS \\ Rheinische Friedrich-Wilhelms-Universität Bonn
}

\title{
Förderung bildungssprachlicher Kompetenzen von Schülerinnen und Schülern im Fach als Teil der Lehrerausbildung
}

\begin{abstract}
:
Developing subject-specific language competence in pupils as an element of teacher training programmes. An example from the module "Teaching immigrant pupils in their second language (German)"

In order to guarantee fairness in learning and access to education regardless of social factors or first language, teachers need to be able to recognize not only the subject-specific, but also the linguistic aspects of their teaching, and develop the necessary competences in their students. This paper explains and illustrates how the module "Teaching immigrant pupils in their second language (German)" at the University of Bonn prepares teachers for this task.
\end{abstract}

\section{Einführung}

Das schlechte Abschneiden deutscher Schülerinnen und Schüler (SuS) in den PISAStudien hat eine große und anhaltende bildungspolitische Debatte ausgelöst, bei der es nicht zuletzt auch um die Chancengerechtigkeit des Bildungssystems in Deutschland geht. Ein zentrales Ergebnis lautet nämlich, dass der Bildungserfolg in keinem anderen OECD-Land so stark von der sozialen Herkunft abhängt wie in Deutschland (vgl. E. Klieme/ C. Artelt/ J. Hartig/ N. Jude/ O. Köller/ M. Prenzel/ W. Schneider/ P. Stanat 2010). Insbesondere geriet die Gruppe der SuS mit Zuwanderungsgeschichte in den Fokus der Diskussion. Doch bei genauerem Hinsehen zeigt sich, dass auch bei SuS ohne Zuwanderungsgeschichte vergleichbar schlechte Ergebnisse erzielt werden, wenn die Merkmale eines tendenziell ungünstigeren sozioökonomischen Status des Elternhauses und eine relative Bildungsferne der Familien vorliegen (vgl. E.D. Klein/ I. van Ackeren 2014; H. Barkowski/ H.-J. Krumm 2010). Beiden Schülergruppen gemeinsam ist eine Diskrepanz zwischen den bestehenden und erwarteten bildungssprachlichen Fähigkeiten, das heißt eine nicht ausreichende Beherrschung des Registers Bildungssprache, wie es „von ,erfolgreichen Schülerinnen und Schülern` erwartet wird“ (I. Gogolin/ Lange 2011: 111). Bei der Ursachensuche wurden unter anderem „Faktoren [genannt], die in der Gestaltung der Schule und des Unterrichts liegen. Dazu gehört die unterlassene Einführung in die schul- und bil- 
dungsrelevanten sprachlichen Fähigkeiten“ (I. Gogolin 2010: 29). Auch Feilke weist darauf hin, dass der adäquate Gebrauch zwar als selbstverständlich von der Schule vorausgesetzt, aber gar nicht gelehrt wird (vgl. H. Feilke 2012: 4). Denn entgegen der Vorstellung, dass sich bildungssprachliche Kompetenz bei SuS im Verlauf der Schulzeit von alleine einstellt, gehen aktuelle bildungssprachliche Konzepte davon aus, dass diese „durch bewusste didaktische Unterstützungsmaßnahmen in enger Verbindung mit Fachunterricht gezielt identifiziert, benannt und fokussiert werden“ (H.-J. Roth/ C. Bainski/ A. Brandenburger/ J. Duarte 2012: 94) müssen. Dies bedeutet, dass Lehrerinnen und Lehrer über die Kompetenz verfügen sollten, die in ihrem jeweiligen Fach verwendete Sprache fördern zu können. Den Ergebnissen einer repräsentativen Umfrage im Auftrag des Mercator-Instituts für Sprachförderung und Deutsch als Zweitsprache aus dem Jahr 2012 zufolge, erkennen tatsächlich auch 70 \% der Lehrerinnen und Lehrer einen Sprachförderbedarf bei ihren SuS, jedoch fühlen sich zwei Drittel der Befragten durch das Studium nicht entsprechend auf diese Aufgabe vorbereitet (vgl. M. Becker-Mrotzek/ B. Hentschel/ K. Hippmann/ M. Linnemann 2012). Auf der bildungspolitischen Ebene plädiert die Beauftragte der Bundesregierung für Migration, Flüchtlinge und Integration, Böhmer im Jahr 2012, dass die „hinsichtlich ihrer Sprachkompetenzen heterogenen Schülerinnen und Schüler [...] ein sehr differenziertes Sprachförderangebot der Schulen und gut ausgebildete Lehrkräfte [erfordern]“ (M. Böhmer 2012: 118) und bekräftigt damit die in einigen Bundesländern bereits erfolgten curricularen Schritte in der Lehreraus- und Weiterbildung. So reformierte das Land Nordrhein-Westfalen im Jahr 2009 das Lehrerausbildungsgesetz (LABG) und führte ein verpflichtendes Modul ein, in dem „Leistungen in Deutsch für Schülerinnen und Schüler mit Zuwanderungsgeschichte (...) für alle Lehrämter zu erbringen“ sind (Ministerium für Schule und Weiterbildung 2015: 2), verbunden mit dem Ziel, angehende Lehrerinnen und Lehrer aller Fächer auf den professionellen Umgang mit sprachlich heterogenen Klassen vorzubereiten. Der vorliegende Artikel beschreibt inhaltliche Aspekte des Pflichtmoduls und illustriert die konkrete hochschuldidaktische Umsetzung an der Rheinischen FriedrichWilhelms-Universität Bonn anhand von ausgewählten Aufgabenbeispielen.

\section{Bildungssprache im Fach}

Der Begriff Bildungssprache findet in dem bereits erwähnten deutschen Bildungsdiskurs der letzten Jahre häufig Verwendung. Die Hamburger Erziehungswissenschaftlerin Ingrid Gogolin und das von ihr mitverantwortete bundesweit geförderte Projekt FörMig zur Erarbeitung von Gesamtkonzepten zur Förderung von Kindern und Jugendlichen mit Migrationshintergrund (vgl. https://www.foermig.unihamburg.de/de.html) rekurrieren dabei auf die Ausführungen des Philosophen und Soziologen Jürgen Habermas (1977). Habermas versteht unter Bildungssprache diejenige Sprache, mit deren Hilfe man sich „mit den Mitteln der allgemeinen Schulbildung ein Orientierungswissen verschaffen [kann]“ (J. Habermas 1977: 345). Die Bildungssprache charakterisiert sich in seinem Verständnis „vor allem durch ein hohes Maß an konzeptioneller Schriftlichkeit (Orientierung an der Schriftsprache) und einen Wortschatz, der ein fachspezifisches Vokabular einschließt“ (K. Beren- 
des/ N. Dragon/ S.Weinert/ B. Heppt/ P. Stanat 2013: 19). In der deutschsprachigen Literatur zum Thema wird Bildungssprache als eigenes Register bezeichnet (vgl. K. Ehlich 1999) und üblicherweise in Abgrenzung zu Alltagssprache dargestellt (vgl. u.a. H. Ortner 2009: 2227; I. Gogolin/ I. Lange 2010: 12; J. Leisen 2010: 46; U. Ohm 2010: 88).

Angeführt wird in diesem Zusammenhang die auf den kanadischen Mehrsprachigkeitsforscher Jim Cummins zurückgehende Unterscheidung von der alltagssprachlichen dialogischen Sprachkompetenz (BICS - Basic Interpersonal Communication Skills) gegenüber der kognitiv-akademischen Sprachkompetenz (CALP Cognitive Language Academic Proficiency) (vgl. J. Cummins 1979). Im Unterschied zur Alltagssprache, die konzeptionell mündlich realisiert wird, zeigt die Bildungssprache konzeptionell schriftliche Merkmale (vgl. P. Koch/ W. Oesterreicher 1997). Im schulischen Kontext kennzeichnet sich die Bildungssprache nach Reich durch „die funktionalen Textsorten des Unterrichts, durch schriftsprachliche Wortwahl und Grammatik, durch differenzierte Bedeutungen“ (H.H. Reich 2013: 9). Gleichzeitig weist sie nach Gogolin und Lange (vgl. I. Gogolin/I. Lange 2010: 12) Überschneidungen mit den Begriffen Schulsprache und Fachsprache auf. Unter Schulsprache versteht Feilke „auf das Lehren bezogene und für den Unterricht $z u$ didaktischen Zwecken gemachte Sprach- und Sprachgebrauchsformen, aber auch Spracherwartungen“ (H. Feilke 2012: 5, Hervorhebung im Original). Als Beispiel nennt er in diesem Zusammenhang die didaktischen Textsorten der Fächer, in diesem Fall die Erörterung, die eigens in der Schule geschrieben wird (vgl. H. Feilke 2012: 5). „Unter Fachsprache versteht man [...] eine spezielle Ausprägung von Sprache, die zur effizienten und präzisen Kommunikation unter Fachleuten dient. Dabei geht es meist um berufsspezifische Sachbereiche und Tätigkeitsfelder[.] [...] Bildungssprache enthält fachsprachliche Elemente, und zwar vor allem im Bereich der Terminologie - dem besonderen Wortschatz der Unterrichtsfächer“ (I. Gogolin/ I. Lange 2010: 12, Hervorhebung im Original). In der folgenden Übersicht werden in der Literatur genannte Merkmale von konzeptioneller Schriftlichkeit in Abgrenzung zur konzeptionellen Mündlichkeit auf vier Ebenen zusammenfassend dargestellt. Eine empirisch fundierte umfassende Beschreibung bildungssprachlicher Merkmale stellt für die deutsche Sprache jedoch noch ein Desiderat dar (vgl. L. Riebling 2013: 132).

Einen Vorschlag, die sprachlichen Anforderungen der Schule näher zu bestimmen, stellen die im Rahmen des FörMig-Projektes erarbeiteten Niveaubeschreibungen Deutsch als Zweitsprache für die Primarstufe und die Sekundarstufe I dar. Ähnlich dem Gemeinsamen Europäischen Referenzrahmen für Sprachen (GER) wird das Kontinuum der sprachlichen Entwicklung hier im Kontext der Schule in sieben Kompetenzbereichen in vier Niveaustufen (I als niedrigste und IV als höchste Stufe) eingeteilt. Die dazu verwendeten Deskriptoren sollen eine kriteriengestützte Einschätzung sprachlichen Handelns von SuS ermöglichen, die als Grundlage pädagogischer Planung im Sinne der durchgängigen Sprachbildung (vgl. I. Gogolin / I. Lange 2010) herangezogen werden kann. Die vier Niveaustufen stellen Bezugsgrößen dar, „durch die die Aneignung des Deutschen als Zweitsprache auf das Deutsche als Bildungssprache hin ausgerichtet ist“ (H.H. Reich 2013: 5). 


\begin{tabular}{|c|c|}
\hline \multicolumn{2}{|r|}{ SCHRIFTLICHKEIT } \\
\hline \multicolumn{2}{|c|}{ Kommunikationsbedingungen } \\
\hline $\begin{array}{l}\text { dialogisch, interaktiv (vertrautes Gespräch, Tele- } \\
\text { fonat mit Freund) }\end{array}$ & monologisch (Vortrag, Referat, Aufsatz) \\
\hline $\begin{array}{l}\text { Vertrautheit der Partner (privates Gespräch) } \\
\text { face-to-face Interaktion }\end{array}$ & $\begin{array}{l}\text { Fremdheit der Partner (Vorstellungsgespräch) } \\
\text { raumzeitliche Trennung }\end{array}$ \\
\hline privat ( Privatbrief, Tagebucheintrag, SMS) & öffentlich (Protokoll, Bericht, Erörterung) \\
\hline spontan & $\begin{array}{l}\text { reflektiert (logische Gliederung, ein dem Gegen- } \\
\text { stand angemessener Textumfang) }\end{array}$ \\
\hline Situationsverschränkung & Situationsentbindung \\
\hline Affektivität & Objektivität \\
\hline \multicolumn{2}{|c|}{ Versprachlichungsstrategien } \\
\hline Prozesshaftigkeit & Vergegenständlichung \\
\hline Vorläufigkeit & Endgültigkeit \\
\hline \multicolumn{2}{|c|}{ Lexikalisch-semantische Merkmale } \\
\hline $\begin{array}{l}\text { undifferenzierte und allgemeine Ausdrücke (rauf } \\
\text { bringen) }\end{array}$ & $\begin{array}{l}\text { differenzierte und abstrahierende Ausdrücke } \\
\text { (nach oben transportieren) }\end{array}$ \\
\hline reduzierter Wortschatz & $\begin{array}{l}\text { Präfixverben, viele untrennbar oder reflexiv } \\
\text { (erhitzen, sich entfalten, sich beziehen) } \\
\text { nominale Zusammensetzungen (Winkelmesser) } \\
\text { und normierte Fachbegriffe (rechtwinklig, Drei- } \\
\text { satz) }\end{array}$ \\
\hline \multicolumn{2}{|c|}{ Syntaktische und textuelle Merkmale } \\
\hline $\begin{array}{l}\text { parataktisch (Aneinanderreihung von Hauptsät- } \\
\text { zen) }\end{array}$ & $\begin{array}{l}\text { hypotaktisch (Konjunktionalsätze, Relativsätze, } \\
\text { erweiterte Infinitive) }\end{array}$ \\
\hline einfacher Satzbau (Subjekt, Prädikat, Objekt) & $\begin{array}{l}\text { explizite Markierungen oder Kohäsion (Textzu- } \\
\text { sammenhang) }\end{array}$ \\
\hline & $\begin{array}{l}\text { unpersönliche Konstruktionen (Passivsätze, } \\
\text { man-Sätze) }\end{array}$ \\
\hline & $\begin{array}{l}\text { Funktionsverbgefüge (zur Explosion bringen, } \\
\text { einer Prüfung unterziehen) }\end{array}$ \\
\hline & $\begin{array}{l}\text { Umfängliche Attribute (der sich daraus ergeben- } \\
\text { de Schluss) }\end{array}$ \\
\hline
\end{tabular}

Abbildung 1. Merkmale konzeptioneller Mündlichkeit/ Schriftlichkeit (nach H. Günther 1997: 67;

G. Kniffka/ G. Siebert-Ott 2007: 20; H.H. Reich 2008; I. Gogolin/I. Lange 2010: 13)

Die Niveaubeschreibungen Deutsch als Zweitsprache für die Sekundarstufe I sind ein Instrument mit dem der sprachliche Kompetenzzuwachs von SuS der Klassen 5 bis 9 strukturiert beobachtet und beschrieben werden kann. Die insgesamt 27 Sprachaneignungsprozesse sind den folgenden sieben Kompetenzbereichen zugeordnet:

A. Weite der sprachlichen Handlungs- und Verstehensfähigkeit
B. Wortschatz
C. Aussprache
D. Lesen
E. Schreiben
F. Grammatik - mündlich und schriftlich 
G. Persönlichkeitsmerkmale (vgl. M. Döll 2013: 11).

Anhand von ausgewählten Niveaubeschreibungen wollen wir die bildungssprachlichen Kompetenzen zur erfolgreichen Teilhabe am Diskurs des Fachunterrichts in der Sekundarstufe I veranschaulichen.

Im Bereich A, der Weite der sprachlichen Handlungs- und Verstehensfähigkeit, wird zwischen vier Sprachaneignungsprozessen unterschieden. In Unterrichtsgesprächen beispielsweise sollen SuS auf der Niveaustufe IV dazu fähig sein, Fachvorträge zu verstehen, Nachfragen dazu zu stellen und sich zusammenhängend zu Fachinhalten zu äußern. Zudem sollen sie Meinungen begründen und Standpunkte vertreten können (vgl. M. Döll / H.H. Reich 2013: 17). Die Wortschatzebene (Bereich B) unterteilt sich in Verstehens-, Mitteilungs-, und Fachwortschatz. Auf der rezeptiven Ebene wird die nachfolgende Zielvorstellung formuliert: „Der Schüler versteht die Begriffe des altersgemäßen gehobenen Aufbauwortschatzes. Dieser Wortschatz umfasst über den Grundwortschatz hinaus Begriffe des Bildungswortschatzes („Schädling“, „emsig“, „erschließen“) und eine Reihe spezieller Fachbegriffe aus schulisch oder außerschulisch relevanten Themenbereichen (z.B. „Halbleiter“, „Isolatoren“, „Fotosynthese“)“ (ebd.: 19, Hervorhebungen im Original). Auf der Mitteilungsebene sieht die Niveaustufe IV vor, dass SuS in der Lage sind, den oben genannten Verstehenswortschatz auch produktiv anzuwenden (vgl.: ebd. 20). Die im Fachunterricht der Schule verwendeten Fachbegriffe und fachlichen Wendungen werden der dritten Kategorie, Fachwortschatz, zugeordnet. Hier sollen SuS auf der obersten Stufe fähig sein, ihnen „bislang unbekannte Fachbegriffe und fachliche Wendungen zu erschließen“ (ebd.: 20, Hervorhebungen im Original). Auch für die Fertigkeiten des schulischen Lesens und Schreibens werden Deskriptoren formuliert. Zur Einschätzung des Leseverstehens bietet das Instrument eine Hilfestellung, um festhalten zu können „in welchem Umfang ein Schüler einem altersgemäßen Text Informationen entnehmen und diese verarbeiten kann“ (ebd.: 22). Auf der Niveaustufe IV kann demnach „[d]er Schüler [...] den Inhalt anspruchsvollerer Texte erfassen. Er kann Schlussfolgerungen ziehen und ist in der Lage, Texte zu bewerten“ (ebd.: 22, Hervorhebungen im Original). Als Ziel der sprachlichen Entwicklung im Bereich der Textproduktion sollen die SuS imstande sein, verständliche schwierige Texte zu verfassen, bei denen die Darlegung komplexer Zusammenhänge verlangt wird (vgl. ebd.: 24).

Deutlich wird, dass die Niveaubeschreibungen lediglich eine Orientierung bieten. Als Grundlage für die Planung eines systematischen Aufbaus von bildungssprachlichen Kompetenzen im Fach wäre jedoch eine weitere Konkretisierung und fachliche Ausdifferenzierung der Anforderungen wünschenswert. Dies gilt sowohl für die allgemeinbildenden Schulen als auch für die Berufsschulen (vgl. Ch. Efing 2012, 2015). Hinzu kommt dass die Anforderungen stetig ansteigen. Gerade im Fachunterricht gelangen SuS mit sprachlichen Defiziten an ihre sprachlichen und fachlichen Grenzen. Dies kann SuS mit Deutsch als Zweitsprache, aber auch Kinder und Jugendliche ohne Zuwanderungsgeschichte betreffen, wenn die bereits oben genannten Merkmale eines tendenziell ungünstigeren sozioökonomischen Status des Elternhauses und eine relative Bildungsferne der Familien vorliegen (vgl. E.D. Klein/ 
I. van Ackeren 2014; H. Barkowski/ H.-J. Krumm 2010). Häufig stellen bereits Aufgabenformulierungen SuS vor sprachliche Herausforderungen und versperren ihnen somit den Zugang zur Erschließung fachlicher Inhalte. Die darin enthaltenen Sprachhandlungsverben, die im schulischen Kontext seit einigen Jahren als Operatoren bezeichnet werden (z.B. Beschreiben, Erklären, Definieren, Erläutern), verlangen sprachliche Ausführungen, die in Aufgaben zwar explizit genannt, aber oft gar nicht oder unzureichend reflektiert und vermittelt werden (vgl. H.J. Vollmer/ E. Thürmann 2010: 111; S. Schmölzer-Eibinger 2013: 27).

Die enge Verknüpfung von Sprache und Fachlernen impliziert bereits mögliche didaktische Schlussfolgerungen für die Gestaltung der Lehr- und Lernprozesse im Kontext der schulischen Bildung. Der Referenzrahmen Schulqualität NRW sieht daher einen bewussten Umgang mit Sprache in allen Fächern und schulischen Handlungsbereichen vor und verweist auf die Notwendigkeit einer systematischen und koordinierten Unterstützung beim Erwerb der Bildungssprache im sprachsensiblen Fachunterricht (vgl. Ministerium für Schule und Weiterbildung 2015a: 33). Da ohne Sprache Wissenserwerb im Fachunterricht nicht gelingen kann, sollen die Sprachstände der SuS „bei der Planung und Gestaltung der unterrichtlichen Prozesse mit dem Ziel berücksichtigt [werden], fachliche Verstehensprozesse zu erleichtern und bildungssprachliche Kompetenzen aktiv zu fördern“ (ebd.: 33). Die Integration von „Sprach- und Fachlernen bedeutet für den Fachunterricht, dass die Aufmerksamkeit der Lehrkräfte nicht nur den Inhalten, sondern auch der Sprache gilt und didaktische Verfahren eingesetzt werden, die inhaltliche Verstehens- und Lernprozesse durch gezielte Spracharbeit unterstützen“ (S. Schmölzer-Eibinger 2013: 32). Im Folgenden möchten wir am Beispiel des Moduls Deutsch für Schülerinnen und Schüler mit Zuwanderungsgeschichte darstellen, wie die Vorbereitung angehender Lehrerinnen und Lehrer auf diese Aufgabe an der Rheinischen Friedrich-Wilhelms-Universität Bonn realisiert wird.

\section{Sprachliche Bildung als Aspekt der Lehrerausbildung}

Das seit 2009 im Bundesland Nordrhein-Westfalen für das Lehramtsstudium verpflichtende Modul „Deutsch für Schülerinnen und Schüler mit Zuwanderungsgeschichte“ (im Folgenden DSSZ-Modul) wird an der Universität Bonn im ersten Jahr der Masterphase als einsemestriges vierstündiges (4 SWS) Seminar umgesetzt. Die auf maximal 30 Teilnehmende begrenzten Seminargruppen setzen sich aus Studierenden des Lehramtes für Gymnasien, Gesamtschulen und Berufskollegs aller 20 in Bonn studierbaren Fächer zusammen. Um der Heterogenität der Seminargruppen adäquat begegnen zu können, wurde das Seminar im Blended Learning-Format konzipiert. Dabei finden 3 SWS als Präsenzveranstaltung und 1 SWS online über die universitäre Lernplattform statt. Die Präsenzanteile dienen einer fachübergreifenden Einführung in die Modulinhalte, durch die Onlineanteile wird eine fachspezifische Differenzierung vorgenommen. Beide Komponenten sind nach dem Prinzip des Blended Learning nicht nur inhaltlich, sondern auch methodisch miteinander verzahnt (vgl. P. Arnold/ L. Kilian/ A. Thillosen/ G. Zimmer, 2015: 142). 
Das DSSZ-Modul gliedert sich in fünf thematische Blöcke, deren Verteilung auf Präsenz- und Onlineanteile in der folgenden Grafik veranschaulicht wird.

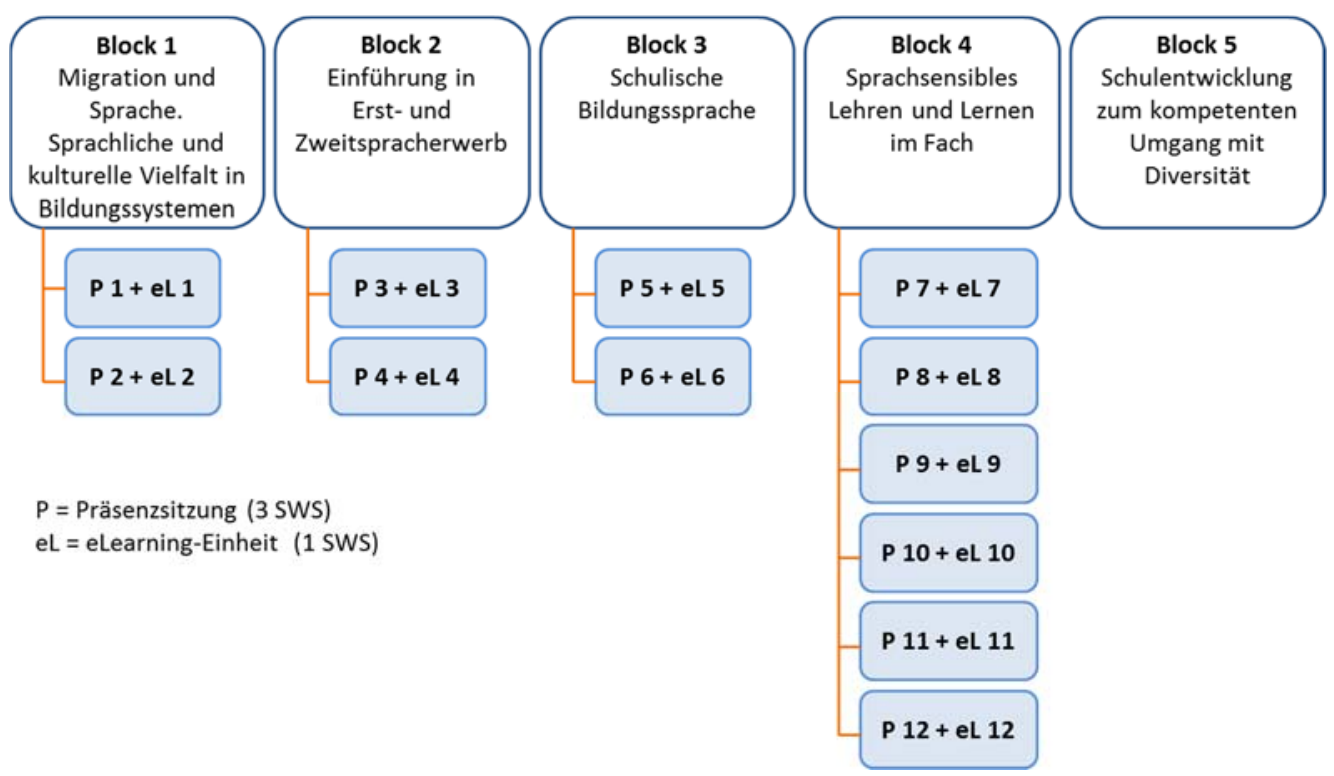

Abbildung 2. Blended Learning-Struktur des Bonner DSSZ-Moduls

Ziel ist es, die angehenden Lehrerinnen und Lehrer auf den kompetenten Umgang mit einer sprachlich heterogenen Schülerschaft vorzubereiten. Dabei sollen neben der Sensibilisierung für die Bedeutung der Sprache für den Bildungserfolg die bildungssprachlichen Anforderungen in der Schule und im eigenen Fach erkannt und analysiert werden. Die Lehramtsstudierenden lernen zudem Diagnoseinstrumente zur Ermittlung des Sprachstandes von SuS kennen und erwerben grundlegende Kompetenzen zur Gestaltung eines sprachsensiblen Unterrichts im Fach.

Nachfolgend stellen wir die in Bonn realisierte hochschuldidaktische Umsetzung vor, indem wir auf Aufgabenbeispiele aus dem Block 3 eingehen, um anhand dieser zu zeigen, wie der im Rahmen des vorliegenden Beitrages beschriebene Themenkomplex der schulischen Bildungssprache im DSSZ-Modul behandelt wird. Dabei haben wir bewusst die Aufgaben bzw. Aufgabenabfolgen gewählt, die die Umsetzung im Präsenzbereich (unter 2.1), im Online-Bereich (unter 2.3) und auch auf der Schnittstelle beider Komponenten (unter 2.2) illustrieren. Die hochschuldidaktische Vorgehensweise zur Vermittlung didaktischer Ansätze des sprachsensiblen Unterrichtens innerhalb des vierten Blocks, Sprachsensibles Lehren und Lernen im Fach, wurde von uns bereits an anderer Stelle fokussiert (vgl. A. Backhaus/ J. Chlebnikow 2017).

\subsection{Aufgabenbeispiel: Wasserkreislauf}

Die Aufgabe Wasserkreislauf bildet einen selbstreflexiv angelegten Einstieg in den 3. Block des DSSZ-Moduls zum Thema schulische Bildungssprache. Das Ziel dieser 
Aktivität besteht in einer Sensibilisierung der Studierenden für sprachliche Anforderungen schulischer Aufgaben.

Zu Beginn der Präsenzveranstaltung werden die Studierenden gebeten, den Wasserkreislauf zu beschreiben (vgl. C. Benholz 2012). Dabei handelt es sich um eine Aufgabe, die im Lehrplan des Geografieunterrichts der 5. bzw. 6. Klasse vorgesehen ist. Die Beschreibung soll in schriftlicher Form umgesetzt werden. Zusätzlich soll der Text in einer Zweitsprache verfasst werden. Bei den meisten Studierenden handelt es sich dabei um Englisch. Zur Unterstützung erhalten die Studierenden die folgende Grafik.

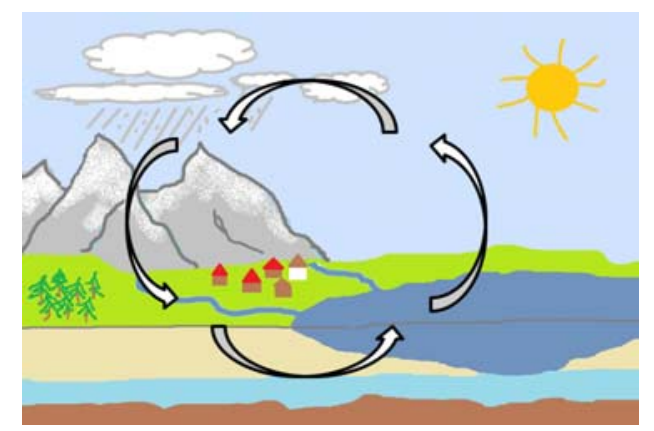

Abbildung 3. Wasserkreislauf

Die anschließende Reflexionsphase fokussiert die Erfahrungen der Studierenden bei der Erstellung des Textes. Sie berichten von fehlenden sprachlichen Mitteln auf der Wort-, Satz,- und Textebene auf die sie bei dem Versuch, den Text schriftsprachlich zu konzipieren, gestoßen sind. Die dabei gewonnenen Erkenntnisse dienen als Ausgangspunkt einer theoretischen Auseinandersetzung mit Anforderungen der schulischen Bildungssprache.

\subsection{Aufgabenbeispiele: Operatoren im Fach}

In den Bildungsstandards und Kernlehrplänen der Fächer sind die Lernziele zumeist ergebnisorientiert formuliert. Die fachlichen Kompetenzen sind dabei an sprachliche Handlungen wie Beschreiben, Begründen, Erklären usw. gebunden und als sog. Operatoren für jedes Fach verbindlich festgelegt. Bei der Betrachtung der schulischen Bildungssprache im Rahmen des DSSZ-Moduls spielt daher auch die Auseinandersetzung mit den Operatoren eine wichtige Rolle. Die hier vorgestellte Aufgabenabfolge beginnt mit einer Online-Aufgabe, die in der nachfolgenden Präsenzveranstaltung fortgeführt wird.

Nach einem thematischen Einstieg in die eLearning-Einheit, bei dem sich die Studierenden mit den Operatorenlisten ihrer jeweiligen Fächer beschäftigt haben, besteht das Ziel der Online-Aufgabe darin, Operatoren in den Aufgabenstellungen der Lehrwerke der eigenen Fächer zu erkennen und zu verstehen, dass gleiche Operatoren in unterschiedlichen Fächern andere kognitive und sprachliche Kompetenzen verlangen können. Dazu sichten die Studierenden zunächst Lehrwerke ihrer Fächer auf der Suche nach Aufgabenstellungen, die einen der folgenden Operatoren bein- 
halten: Beschreiben, Zusammenfassen, Vergleichen, Erklären, Begründen, Stellung nehmen, Bewerten und Diskutieren. Dabei handelt es sich um Operatoren, die in allen Fächern vorkommen. Danach ordnen sie die gefundenen Beispiele in ein gemeinsames Wiki unter den entsprechenden Operatorenkategorien ein.

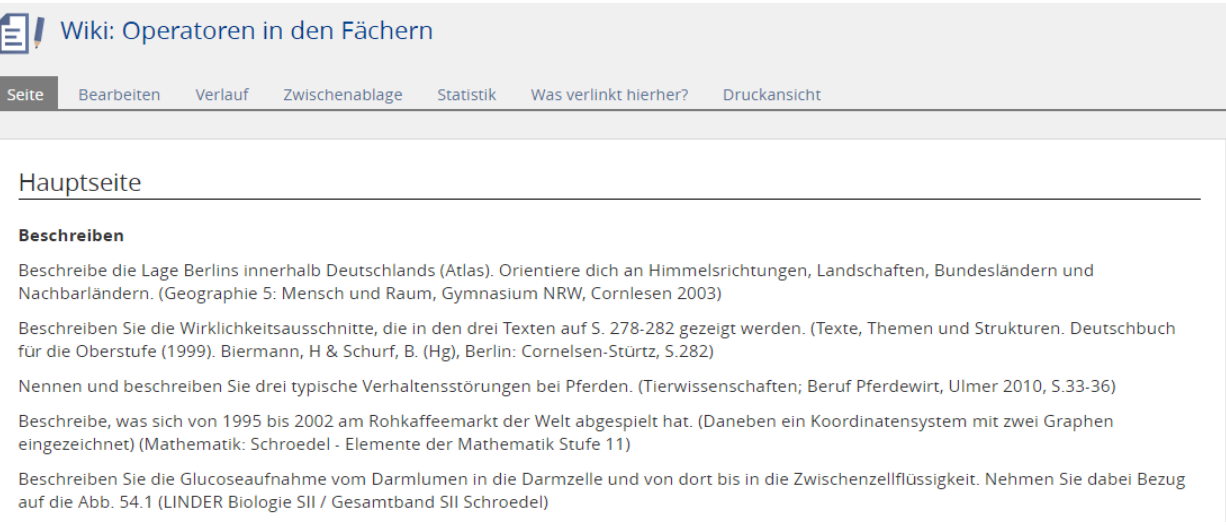

Abbildung 4. Ergebnisausschnitt aus dem Wiki: Operatoren in den Fächern

Abschließend vergleichen die Studierenden die zusammengetragenen Aufgabenstellungen und notieren erste Beobachtungen für die Weiterarbeit.

Die Ergebnisse aus der Online-Aufgabe bilden die Grundlage für die daran anknüpfende Aufgabe der folgenden Präsenzsitzung. Die Studierenden sollen erkennen, welche sprachlichen Anforderungen die von ihnen ausgewählten Aufgabenstellungen enthalten. Dazu finden sie sich in einem ersten Schritt in Fachgruppen zusammen und analysieren, welcher Wortschatz und welche sprachlichen Strukturen in den Anweisungen vorkommen. In einem zweiten Schritt arbeiten die Studierenden individuell und führen exemplarisch eine der Aufgabenstellungen schriftlich aus. In der letzten Phase treffen sie sich erneut in der Fachgruppe und wiederholen die sprachliche Analyse, nun bezogen auf ihre erstellten Texte. Auf diese Weise nähern sich die Studierenden den von ihnen implizit vorausgesetzten Standards der Ergebnisse und reflektieren ihren fach- und bildungssprachlichen Erwartungshorizont.

\subsection{Aufgabenbeispiele: Bildungssprache im Fach}

Bei dem letzten hier beschriebenen Beispiel handelt es sich um eine Aufgabenabfolge zum Thema Bildungssprache im Fach. Die eLearning-Einheit beginnt (wie alle eLearning-Einheiten) mit einer Kurzdarstellung der Inhalte, wie die folgende Abbildung illustriert.

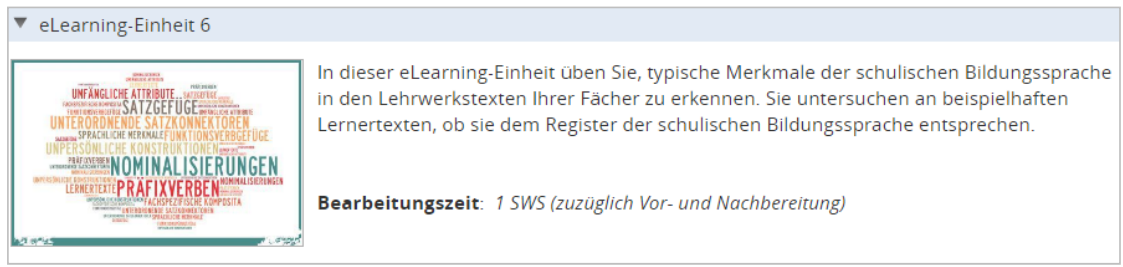

Abbildung 5. Einstieg in eLearning-Einheit 6 
Die eLearning-Einheit setzt sich aus drei Online-Aufgaben zusammen, die darauf zielen, dass Studierende bildungssprachliche Merkmale kennen lernen, sie in ihrem Fach erkennen können und für fachliche vs. sprachliche Schwierigkeiten sensibilisiert werden.

In der ersten Online-Aufgabe setzen sich die Studierenden mit den bildungssprachlichen Merkmalen (vgl. Reich 2008) auseinander und sollen diese anhand einer vorgegebenen Liste in den Lehrwerkstexten ihrer Fächer identifizieren. Die Studierenden werden gebeten, zwei Sätze auszuwählen, in denen mindestens eins der folgenden Merkmale vorkommt: Fachspezifische Komposita, Nominalisierungen, Präfixverben, Satzgefüge, unterordnende Satzkonnektoren, unpersönliche Konstruktionen, Funktionsverbgefüge oder umfängliche Attribute. Diese sollen unter der entsprechenden Kategorie in einem gemeinsamen Wiki eingetragen werden.

Wiki: Schulische Bildungssprache

Seite Bearbeiten Verlauf Zwischenablage Statistik Was verlinkt hierher? Druckansicht
Hauptseite

Umfängliche Attribute (z. B. die nach oben offene Richter-Skala, der sich daraus ergebende Schluss)

Das Vertrauensintervall zu einer relativen Häufigkeit h enthält alle Wahrscheinlichkeiten $p$, die die mit einer beobachteten relativen Hāufigkeit $h$ mit einer vorgegebenen Sicherheitswahrscheinlichkeit (Vertrauensniveau) $\beta$ vereinbar sind. (Mathematik)

Das für den Prozess nach Solvay benötigte Kohlenstoffdioxid wird durch das Brennen von Kalkstein gewonnen. (Chemie)

"Erläutern Sie die zu sehenden Emotionen der Menschen in Bild M8 basierend auf Ihrem Vorwissen zur deutsch-österreichischen Geschichte seit 1815." (Geschichte)

Abbildung 6. Ergebnisausschnitt aus dem Wiki: Schulische Bildungssprache

Die zweite Online-Aufgabe ist binnendifferenzierend für Mathematik, Naturwissenschaften und Gesellschaftswissenschaften gestaltet und fokussiert die jeweiligen spezifischen sprachlichen Merkmale dieser Fächergruppen. Die Aufgabe der Studierenden besteht darin, den fachspezifischen sprachlichen Merkmalen auf Wort-, Satz, und Textebene vorgegebene Beispiele zuzuordnen. Auf diese Weise werden die auf metasprachlicher Ebene benannten Merkmale konkretisiert und für die Studierenden nachvollziehbarer. Die Zuordnungsübung mit automatisiertem Feedback kann beliebig oft wiederholt werden. 
Test: Sprache in den Fächern (Naturwissenschaften)

Zur Surbete:

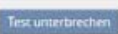

Frage 1 von 3-Merkmale auf der Wortebene (Naturwissenschaften) (10 Punkte)

Ordnen Sie die Beispiele den sprachlichen Mitteln zu

\begin{tabular}{|c|}
\hline $\begin{array}{c}\text { Fachspezifische Abkcurzungen und } \\
\text { Symbole }\end{array}$ \\
\hline $\begin{array}{c}\text { Mit Vorsiben gebildete Fachworter } \\
\text { (Nomen und Verberi) }\end{array}$ \\
\hline $\begin{array}{l}\text { Fachliche Adjektive. Typische Endsilben } \\
\text { sind dabei: -haltig, -reich, -arm, -los, -fest }\end{array}$ \\
\hline $\begin{array}{c}\text { Haufig verwendete Verben (auch mit } \\
\text { festen Prapositionen) }\end{array}$ \\
\hline $\begin{array}{c}\text { Worter, die alltagssprachlich bekannt } \\
\text { sind, die aber im Fachkontext eine } \\
\text { andere Bedeutung haben }\end{array}$ \\
\hline \hline $\begin{array}{c}\text { Fachliche Kollokationen und } \\
\text { Funktionsverbgefoge }\end{array}$ \\
\hline \hline Fachbegriffe aus und mit Eigennamen \\
\hline
\end{tabular}

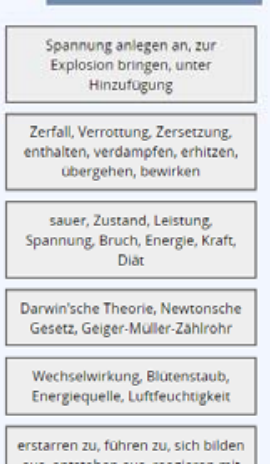

Abbildung 7. Ausschnitt aus der Zuordnungsübung: Sprache in den Fächern

In der diese Sequenz abschließenden dritten Online-Aufgabe arbeiten die Studierenden mit authentischen Schülertexten, die im Kontext unterschiedlicher Fächer entstanden sind. Das Ziel dieser Aufgabe ist, die sprachliche bzw. inhaltliche Angemessenheit der Schülerproduktionen zu reflektieren. Dazu sichten die Studierenden zunächst alle Schülertexte, die in zweifacher Version (Original und Abschrift) zur Verfügung

gestellt werden und posten anschließend im Forum ihre persönliche Einschätzung. Die Studierenden können auf die Beiträge von anderen Bezug nehmen, wie das folgende Beispiel illustriert.

\section{Physik 2}

In Bezug auf den Inhalt würde ich sagen, dass der Schüler die grobe Aussage des ersten Newtonschen Gesetzes verstanden hat, dies aber inhaltlich nicht der Art und Weise entspricht, wie es im Erwartungshorizont stehen würde. Eventuell liegt es aber auch einfach an sprachlichen Schwierigkeiten, die einer präzisen Antwort im Wege stehen. Beispielsweise fehlen dem Schüler feste Muster zur Textgestaltung. Man könnte den Text bspw. folgendermaßen beginnen: "Ein Beispiel für das erste Newtonsche Gesetz...". Generell lässt sich aber sagen, dass der Text trotz Rechtschreib- und Grammatikfehlern in seiner Aussage verständlich ist.

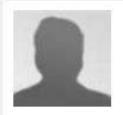

\section{Physik 2}

Ich kann Frau nur zustimmen, dass der betreffende Schüler das Newtonsche Gesetz in seinen wesentlichen

Grundzügen verstanden zu haben scheint, dieses Wissen jedoch nicht angemessen sprachlich umsetzen konnte. Schön ist der Vorschlag fester "Textmuster", welche das Formulieren für den Anfang erleichtern können. Mit ein wenig Hilfestellung lassen sich die kleinen sprachlichen Ungenauigkeiten sehr wahrscheinlich einfach losen. - Da ich weder Physikerin bin, noch mein Grundwissen in Physik annähernd an das Newtonsche Gesetz heranreicht, kann ich inhaltlich gesehen die Aufgabenlösung nicht adäqut beurteilen und überlasse dies gerne meinen Physik-Kommillitonen.

Abbildung 8. Ergebnisausschnitt aus dem Forum: Lernertexte 


\section{Ausblick}

Das Ziel dieses Beitrags war es, am Beispiel des Moduls Deutsch für Schülerinnen und Schüler mit Zuwanderungsgeschichte zu zeigen, wie angehende Lehrerinnen und Lehrer auf den Aspekt der bildungssprachlichen Förderung von SuS im Fach vorbereitet werden können.

Die Evaluationsergebnisse der hochschuldidaktischen Umsetzung des DSSZModuls an der Rheinischen Friedrich-Wilhelms-Universität Bonn zeigen eine große Zustimmung der Studierenden zum gewählten seminardidaktischen Ansatz (vgl. A. Backhaus / J. Chlebnikow, 2017). Die Frage, ob die vermittelten Kompetenzen in der späteren beruflichen Praxis im Fachunterricht Anwendung finden, bleibt noch offen und Bedarf einer Klärung, die im Rahmen einer längerfristig angelegten empirischen Studie wünschenswert wäre.

\section{Bibliographie}

Arnold, P./ L. Kilian/ A. Thillosen/ G. Zimmer (2015), Handbuch E-Learning. Lehren und Lernen mit digitalen Medien. Bielefeld.

Backhaus, A./ J. Chlebnikow (erscheint 2017), „In der Sprache liegt die Würze“Sprachsensibel unterrichten im Fach Ernährungs- und Hauswirtschaftswissenschaften. Ein Blended Learning Konzept für die Lehrerausbildung. In: E. Terrasi-Haufe/ A. Börsel (Hrsg.), Sprache und Sprachbildung in der Beruflichen Bildung. Münster.

Becker-Mrotzek, M./ B. Hentschel/ K. Hippmann/ M. Linnemann (2012), Sprachförderung an deutschen Schulen - die Sicht der Lehrerinnen und Lehrer. Köln. (URL http://www.mercator-institut-sprachfoerderung.de/fileadmin/user_upload/ Lehrerumfrage_Langfassung_final_30_05_03.pdf) [letzter Zugriff 02.01.2017].

Benholz, C (2012), Förderung bei Mehrsprachigkeit in Schule und GanzTag Chancen und Voraussetzungen. Vortrag für das Regionale Bildungsnetzwerk Märkischer Kreis. (URL https://app-mb.lvr.de/rbn/img/26/6c041ab0-8467-41468191-8d55e5221e2c.pdf). [letzter Zugriff 19.01.2017].

Berendes, K./ N. Dragon/ S. Weinert/ B. Heppt/ P. Stanat (2013), Hürde Bildungssprache? Eine Annäherung an das Konzept „Bildungssprache“ unter Einbezug aktueller empirischer Forschungsergebnisse. In: A. Redder/ S. Weinert (Hrsg.), Sprachförderung und Sprachdiagnose. Interdisziplinäre Perspektiven. Münster, 17-41.

Böhmer, M. (2012), 9. Bericht der Beauftragten der Bundesregierung für Migration, Flüchtlinge und Integration über die Lage der Ausländerinnen und Ausländer in Deutschland. Berlin. (URL https://www.bundesregierung.de/ Content/Infomaterial/BPA/IB/2012-12-18-9-_Lagebericht.pdf?_blob $=$ publicationFile\&v=10). [letzter Zugriff 02.01.2017].

Cummins, J. (1979), Cognitive/ academic language proficiency, linguistic interdependence, the optimum age question and some other matters. In: Working Papers on Bilingualism, 19, 121-129. 
Cummins, J. (2006), Sprachliche Interaktionen im Klassenzimmer: Von zwangsweise auferlegten zu kooperativen Formen der Machtbeziehungen. In: P. Mecheril/ Th. Quehl (Hrsg.), Die Macht der Sprachen. Englische Perspektiven auf die mehrsprachige Schule. Münster, 36-62.

Döll, M. (2013), Das Instrument „,Niveaubeschreibungen Deutsch als Zweitsprache für die Sekundarstufe I" Aufbau und Handhabung, Entwicklung, Erprobung und empirische Evaluation, Einführung der Lehrkräfte in die Anwendung. In: Sächsisches Bildungsinstitut (Hrsg.), Niveaubeschreibungen. Deutsch als Zweitsprache für die Sekundarstufe I. Dresden. 11-15.

Döll, M./ Reich, H.H. (2013), Niveaubeschreibungen Deutsch als Zweitsprache für die Sekundarstufe I. In: Sächsisches Bildungsinstitut (Hrsg.), Niveaubeschreibungen. Deutsch als Zweitsprache für die Sekundarstufe I. Dresden.

Efing, Ch. (2012): Sprachliche oder kommunikative Fähigkeiten - was ist der Unterschied und was wird in der Ausbildung verlangt. In: „BWP“ 2, 6-9.

Efing, Ch. (2015): Berufsweltbezogene kommunikative Kompetenz in Erst- und Fremdsprache - Vorschlag einer Modellierung. In: Ch. Efing (Hrsg.), Sprache und Kommunikation in der beruflichen Bildung. Frankfurt a. M., 17-46.

Ehlich, K. (1999), Alltägliche Wissenschaftssprache. In: Info DaF, 26(1), 3-24.

Feilke, H. (2012), Bildungssprachliche Kompetenzen - fördern und entwickeln. In: Praxis Deutsch, 233/2012, 4-13. FörMig-Kompetenzzentrum (URL https://www.foermig.uni-hamburg.de/de.html)

[letzter Zugriff 03.01.2016].

Gogolin, I. (2010), Bildungssprache. In: H. Barkowski/ H.-J. Krumm (Hrsg.), Fachlexikon Deutsch als Fremd- und Zweitsprache. Tübingen, 29.

Gogolin, I./ I. Lange (2010), Durchgängige Sprachbildung. Eine Handreichung. Münster.

Gogolin, I./ I. Lange (2011), Bildungssprache und Durchgängige Sprachbildung. In: S. Fürstenau/ M. Gomolla (Hrsg.), Migration und schulischer Wandel: Mehrsprachigkeit. Wiesbaden, 107-127.

Günther, H. (1997), Mündlichkeit und Schriftlichkeit. In: H. Balhorn/ H. Niemann (Hrsg.), Sprachen werden Schrift. Lengwil, 64-73.

Habermas, J. (1977), Umgangssprache, Wissenschaftssprache, Bildungssprache. In: J. Habermas, Kleine politische Schriften (I-IV). Frankfurt a.M., 340-363.

Klein, E.D./ I. van Ackeren (2014), Woher und Wohin. Soziale Herkunft und Bildungserfolg. Zentrale Grundlagen und Befunde der Schulleistungsvergleichsstudien. Eine Expertise von Prof. Dr. Isabell van Ackeren und Dr. Esther Dominique Klein im Auftrag der Wübben Stiftung.

Klieme, E./ C. Artelt/ J. Hartig/ N. Jude/ O. Köller/ M. Prenzel/ W. Schneider/ P. Stanat (Hrsg.) (2010), PISA 2009. Bilanz nach einem Jahrzehnt. Münster.

Kniffka G./ G. Siebert-Ott (2007), Deutsch als Zweitsprache. Lehren und lernen. Paderborn.

Koch, P./ W. Oesterreicher (1997), Schriftlichkeit und Sprache. In: H. Günther/ O. Ludwig (Hrsg.), Schrift und Schriftlichkeit. Ein interdisziplinäres Handbuchinternationaler Forschung, Halbband 1. Berlin, 587-604. 
Leisen, J. (2010), Handbuch Sprachförderung im Fach. Sprachsensibler Fachunterricht in der Praxis. Bonn.

Ministerium für Schule und Weiterbildung (2015), Gesetz über die Ausbildung für Lehrämter an öffentlichen Schulen (Lehrerausbildungsgesetz - LABG) Vom 12. Mai 2009. (https://www.schulministerium.nrw.de/docs/Recht/LAusbildung/ LABG/LABGNeu.pdf). [letzter Zugriff 02.01.2017].

Ministerium für Schule und Weiterbildung (2015a), Referenzrahmen Schulqualität NRW. (URL http://www.schulentwicklung.nrw.de/e/upload/referenzrahmen/ download/Referenzrahmen_Veroeffentlichung.pdf). [letzter Zugriff 02.01.2017].

Ohm, U. (2010), Von der Objektsteuerung zur Selbststeuerung: Zweitsprachenförderung als Befähigung zum Handeln. In: B. Ahrenholz (Hrsg.), Fachunterricht und Deutsch als Zweitsprache. Tübingen, 87-105.

Ortner, H. (2009), Rhetorisch-stilistische Eigenschaften der Bildungssprache. In: U. Fix/ A. Gardt/ J. Knape (Hrsg.), Rhetorik und Stilistik, Halbband 2. Berlin/ New York, 2227-2240.

Reich H.H. (2008), Materialien zum Workshop „Bildungssprache“. Unveröffentlichtes Schulungsmaterial für die FörMig-Weiterqualifizierung ,Berater(in) für sprachliche Bildung, Deutsch als Zweitsprache“. In: I. Gogolin/ I. Lange (2010), Durchgängige Sprachbildung. Eine Handreichung. Münster.

Reich, H.H. (2013), Zur Einführung in die „Niveaubeschreibungen Deutsch als Zweitsprache für die Sekundarstufe I". In: Sächsisches Bildungsinstitut (Hrsg.), Niveaubeschreibungen. Deutsch als Zweitsprache für die Sekundarstufe I. Dresden, 5-9.

Riebling, L. (2013), Heuristik der Bildungssprache. In: I. Gogolin/ I. Lange/ I. Michel/ H.H. Reich (Hrsg.), Herausforderung Bildungssprache - und wie man sie meistert. Münster, 106-135.

Roth, H.-J./ C. Bainski/ A. Brandenburger/ J. Duarte (2012). Inclusive Academic Language Training. Das europäische Kerncurriculum zur durchgängigen bildungssprachlichen Förderung (EUCIM-TE). In: E. Winters-Ohle/ B. Seipp/ B. Ralle (Hrsg.), Lehrer für Schüler mit Migrationsgeschichte. Münster, 93-114.

Schmölzer-Eibinger, S. (2013), Sprache als Medium des Lernens im Fach. In: M. Becker-Mrotzek/ K. Schramm/ E. Thürmann/ H.J. Vollmer (Hrsg.), Sprache im Fach. Sprachlichkeit und fachliches Lernen. Münster, 25-40.

Vollmer, H. J./ E. Thürmann (2010), Zur Sprachlichkeit des Fachlernens. In: B. Ahrenholz (Hrsg.), Fachunterricht und Deutsch als Zweitsprache. Tübingen, 107-132. 